\title{
Host genotype changes bidirectional to unidirectional cytoplasmic incompatibility in Nasonia longicornis
}

\author{
R Raychoudhury ${ }^{1}$ and JH Werren \\ Department of Biology, University of Rochester, Rochester, NY, USA
}

\begin{abstract}
Wolbachia are the most abundant maternally inherited endosymbionts of insects and cause various reproductive alterations in their hosts. One such manipulation is cytoplasmic incompatibility $(\mathrm{Cl})$, which is a sperm-egg incompatibility typically resulting in zygotic death. Nasonia longicornis (Hymenoptera: Pteromalidae) has an A supergroup and two closely related $B$ supergroup Wolbachia infections. The B supergroup bacteria co-diverged in this host genus. Both triple ( $w \mathrm{Nlo}-$ $\mathrm{nA} w$ NlonB1 $w$ NlonB2) and double infections ( $w$ NlonA $w$ NlonB1, $w$ NlonA $w$ NlonB2) have been obtained from the field. In the present study, $\mathrm{Cl}$ was determined among the three Wolbachia types in different host genetic backgrounds. Results show that host genetic background determines whether bidirectional $\mathrm{Cl}$ or unidirectional $\mathrm{Cl}$ occurs between the two closely related $\mathrm{B}$
\end{abstract}

group Wolbachia. Results show that the wNlonB1-infected males are bidirectionally incompatible with $w$ NlonB2 in their 'native' nuclear genetic background, whereas $w$ NlonB1 males are compatible with $w \mathrm{NlonB2}$ in two other $N$. Iongicornis genetic backgrounds, resulting in unidirectional $\mathrm{Cl}$. In contrast, wNlonB2-infected males are incompatible with $w$ NlonB1 females in all three host genetic backgrounds. These changes in incompatibility are not due to the loss of the bacteria. We hypothesize that a repressor gene for sperm modification by wNlonB1 is segregating in $N$. longicornis populations. The relevance of these findings to the potential role of Wolbachia in host-reproductive divergence and speciation is discussed.

Heredity (2012) 108, 105-114; doi:10.1038/hdy.2011.53; published online 27 July 2011

Keywords: Wolbachia; Nasonia; co-divergence; host genotype; cytoplasmic incompatibility

\section{Introduction}

Wolbachia are one of the most abundant endosymbionts of arthropods and nematodes, infecting about $66 \%$ of all terrestrial arthropods (Hilgenboecker et al., 2008). The principal mode of transmission across generations is through the infected female cytoplasm, where the bacteria are transmitted from the maternal ovaries to the eggs (Werren, 1997; Stouthamer et al., 1999; Stevens et al., 2001; Werren et al., 2008). As they are maternally transmitted and are endosymbiotic, Wolbachia are selected to increase their fitness through the transmitting sex, that is, infected females. This is achieved either by mutualism or by manipulating the host-reproductive machinery to produce more infected females relative to the non-transmitting sex (males). Some of the mechanisms by which Wolbachia achieve this are killing of infected males, induction of parthenogenesis in infected females, feminization of genetic males and cytoplasmic incompatibility (CI) (reviewed in Werren et al., 2008). As a result, Wolbachia-induced host-reproductive manipulation has profound implications for many fundamental

Correspondence: Dr R Raychoudhury, Department of Biology, University of Rochester, 60 crittenden boulevard, apartment 602, Rochester, New York 14627, USA.

E-mail: rraychou@mail.rochester.edu

${ }^{1}$ Current address: Department of Entomology, Purdue University, 901

West State Street, West Lafayette, IN 47907-2089, USA.

Received 23 September 2010; revised 29 April 2011; accepted 31 May

2011; published online 27 July 2011 biological processes, such as sex determination (Rigaud et al., 1997; Werren and Beukeboom, 1998), sexual selection (Jiggins et al., 2000; Koukou et al., 2006), speciation (Laven, 1959; Breeuwer and Werren, 1990; Bordenstein et al., 2001; Jaenike et al., 2006) and organization of host genome structure (Kondo et al., 2002b, Dunning-Hotopp et al., 2007).

Among the various Wolbachia-induced phenotypes, CI seems to be the most common and has received the most attention. According to the 'modification-rescue' model of CI (Werren, 1997), Wolbachia 'modify' the sperm of an infected male and the female must also be infected with the same bacteria to 'rescue' this particular modification. If the female is uninfected or infected with a Wolbachia of a different CI type, then this particular 'modification' cannot be 'rescued' and CI is expressed. When CI occurs reciprocally between two different Wolbachia strains, they are referred to as bidirectionally incompatible. Apparent 'non-functional' and partially functional (for example, modification-deficient but rescue-competent) CI types are also known (Giordano et al., 1995; Hoffmann et al., 1996; Bourtzis et al., 1998; Charlat et al., 2003; Zabalou et al., 2008). Presence of more than one incompatibility type has been found in a number of different insects including mosquitoes, fruit flies, beetles and wasps (Laven, 1959; Breeuwer and Werren, 1990; O'Neill and Karr, 1990; Montchamp-Moreau et al., 1991; PerrotMinnot et al., 1996; Bordenstein et al., 2001). Bidirectional $\mathrm{CI}$ (bi-CI) has attracted particular attention because of its potential role in preventing gene flow between two 
different incompatibility types and thus contributing to reproductive isolation and speciation (Breeuwer and Werren, 1990; Werren, 1998; Bordenstein et al., 2001; Bordenstein, 2003; Telschow et al., 2002, 2005a, b).

Host genotypes can influence Wolbachia density and phenotypes (reviewed in Jaenike, 2009). In the haplodiploid wasp Nasonia, there are two distinct types of CI: conversion of diploid fertilized eggs to haploid males and mortality of fertilized eggs. With introgression of inter-specific genotypes, Bordenstein et al. (2003) showed that the expression of these two types of CI is controlled by host genotype. Sinkins et al. (2005) showed that introgression into a common genetic background can alter bidirectional to unidirectional CI (uni-CI) in Culex quinquefasciatus. Host genotype can also transform Wolbachia induced phenotypes, for example, converting CI to male-killing (Jaenike, 2007) and vice-versa (Hornett et al., 2010). Effects of host genotype on Wolbachia infection density are well established. In the adzuki bean beetle, Callosobruchus chinensis, which is infected with multiple Wolbachia strains, the density of the bacteria is controlled by both intra-specific host genotype, as well as other co-infecting Wolbachia strains (Kondo et al., 2005). In the wasp Leptopilina heterotoma, Wolbachia densities vary based on intra-specific genotypes of two divergent laboratory cultures (Mouton et al., 2007). The mosquito Culex pipiens has a polymorphism for an allele conferring insecticide resistance (Berticat et al., 2002). Mosquitoes carrying the resistant allele have higher Wolbachia densities compared with the ones carrying the susceptible allele (Duron et al., 2006). The role of host genotype in regulating Wolbachia densities has also been empirically established, by transinfection experiments, at an inter-specific level (Ikeda et al., 2003), and at the intra-specific level in Drosophila (Clark et al., 2003).

The parasitoid wasp genus Nasonia has been used extensively in Wolbachia research. It is a genus of four closely related species (Raychoudhury et al., 2010a) that harbor 11 different Wolbachia infections (Raychoudhury et al., 2009). The four species-N. vitripennis, N. longicornis, N. giraulti and $N$. oneida-are infected with multiple Wolbachia infections belonging to supergroups $\mathrm{A}$ and $\mathrm{B}$. N. longicornis has two infections from the B supergroup (named $w$ NlonB1 and $w$ NlonB2) and one from the A (wNlonA) supergroup (Raychoudhury et al., 2009). Moreover, these two B supergroup infections occur as a polymorphism in the field where host strains infected with $w$ NlonB1 $w$ NlonA, $w$ NlonB2 $w$ NlonA and $w$ NlonB1 $w$ NlonB2 $w$ NlonA have been found (Raychoudhury et al., 2009).

The B supergroup Wolbachia in N. longicornis and $N$. giraulti show one of the few documented cases of hostWolbachia co-divergence. The $w$ NlonB1 and $w$ NlonB2 infections are estimated to have diverged $\sim 1.5$ million years ago in the most recent common ancestor of N. longicornis and N. giraulti (Raychoudhury et al., 2009). Since most Wolbachia infections are transient, within a particular host species, over an evolutionary time scale (Vavre et al., 1999; Baldo et al 2006b; Frost et al., 2010), the $N$. longicornis system provides an opportunity to study the long-term effects of Wolbachia-host association.

In the present study we investigate the level and type of CI between these strains, specifically focusing on the host strains with the Wolbachia genotype of $w$ NlonB1wNlonA and $w$ NlonB2wNlonA. We ask the following questions: (1) Do the closely related $w$ NlonB1 and $w$ NlonB2 cause CI against each other?, (2) Do the closely related $w$ NlonB1 and $w$ NlonB2 cause CI against $w$ NlonA? and (3) Are there effects of host genotype on CI?

\section{Materials and methods}

\section{Nomenclature and strains used}

The preferred method of denoting a particular host strain and its Wolbachia infection in Nasonia is to indicate its supergroup, as well as its host genotype. For example, [wNlonB1 $w$ NlonA]L indicates that the host strain is $N$. longicornis, which has two infections, one each from the two supergroups of A and B. In the present study we only use different $N$. longicornis host strains and their associated Wolbachia. Therefore, we have simplified the nomenclature of the bacterial infections by removing the species name, but have included host genotype information. Moreover, as the effects of host genotype have been found to be important for the expression of CI, we have also indicated the source of the bacterial infections. Five host genetic backgrounds (UT1, UT2, UT3, UT4 and CA) have been used and the particular Wolbachia infections from these genetic backgrounds have been designated as a subscript. For example, $(w \mathrm{~A} w \mathrm{~B} 1)_{\mathrm{UT} 1}$ indicates that the strain has two Wolbachia infections and were originally obtained from the UT1 genetic background. The host genetic background is indicated in brackets at the end of the strain designation. For example, (wAwB1) $)_{\mathrm{UT} 1}[U T 1]$ indicates that these Wolbachia infections have UT1 host genetic background. Most of these strains were introgressed in different genetic backgrounds to test for the effect of host genotype on CI, and the designations change accordingly.

Many of the strains were treated with antibiotics to test the effects of host genotype on the crosses in the absence of Wolbachia infections. The nomenclature of cured strains is indicated by 0 followed by the strain origin in brackets, for example, 0[UT1] for the cured strain from the UT1 host genetic background. The different strains used, along with the new nomenclature, are detailed in Supplementary Table S1.

A confounding factor for the determination of the CI relationship between $w$ NlonB1 and $w$ NlonB2 is that they are present as double and triple infections along with the wNlonA Wolbachia. Repeated efforts to make single B-infected strains failed, probably because of their low titer compared with the $w$ NlonA. This was consistent with previous attempts by Bordenstein and Werren (2007). But fortunately we had access to a single $w$ NlonA-infected strain, $(w \mathrm{~A})_{\mathrm{UT} 4}[$ [UT4] obtained by Bordenstein and Werren (2007) from a double-infected host strain (Supplementary Table S1). This strain was used to distinguish the effects of the A supergroup infection in the crosses. To establish that the A infections in all the host strains are the same Multi Locus Sequence Type (MLST) (Baldo et al., 2006b), we amplified and sequenced the A MLST loci from each of the strains. No variation was found in any of the six MLST genes from the four host strains (Raychoudhury et al., 2009). Similarly, all the six MLST loci were amplified from the three strains with B supergroup infections and no 
sequence difference were found between strains for $w$ NlonB1 or wNlonB2 (Raychoudhury et al., 2009).

\section{Introgression lines}

To investigate the effect of host genetic background, introgression lines were produced for most of these strains, where the bacterial genotypes were backcrossed in a different host genetic background. The crosses were done for six generations, whereby, theoretically at least, $98 \%$ of the native genome was replaced by the new host genome. Thus, when (wAwB1) $)_{\mathrm{UT1}}[U T 1]$ females were backcrossed with males from the 0[UT2] strain for six generations it produced the strain $(w \mathrm{~A} w \mathrm{~B} 1)_{\mathrm{UT1}}[$ [UT2]. To make these introgression lines, five females were initially chosen with a particular bacterial polymorphism and mated to the uninfected males with the desired host genotypes. Mating was observed and these females were individually hosted for 2 days. Then the DNA was extracted to check for the presence of the desired bacterial polymorphisms with specific primers. The progeny of all the females with the desired bacterial polymorphism were mixed together. Five virgin females were randomly chosen from this pooled set of offspring to start the next generation of introgression and this was continued for a further five generations. Thus, there were five different isofemale lines for each set of introgression. These strains were allowed to sib-mate for two generations before being used for the experimental crosses. Out of the five sets of strains for each particular introgression only one strain was used for the crosses.

\section{Crossing design}

All crosses were set up as single-pair mating between a virgin male and a virgin female within 2 days of emergence. Individual pairs were observed until they mated, which was mostly within the first $5 \mathrm{~min}$ of observation. They were kept overnight at $25^{\circ}$ with a drop of honey for feeding. The next day the males were discarded and the females were provided with two Sarcophaga bullata hosts. The females were allowed to parasitize the hosts for $48 \mathrm{~h}$, upon which they were discarded and the progeny was allowed to emerge. Upon the death of the progeny they were scored for sex ratio and total family size. To measure levels of cytoplasmic incompatibility, male and female offspring numbers are compared with the females of a strain who are mated to males of different Wolbachia infection types versus to uninfected males. This is a valid approach because females do not alter the number of eggs they lay based on their mating status (Breeuwer and Werren, 1995), making comparisons in the number of male and female progeny produced by females of the same strain who are mated to males of an infection type relative to uninfected males a reliable index of offspring mortality and sex conversion due to cytoplasmic incompatibility. Individual crosses where the females died before the $48 \mathrm{~h}$ of parasitization or produced diapause larvae were not counted and were discarded from the analysis. All of the crosses were repeated and were consistent with each other. We have presented all the data from the two trials in the online Supplementary Material (Supplementary Tables S2 and S3) and have presented a summary of these crosses (Table 1) and figures for the first trial of each cross in the main text. The analysis presented in the main text is based on the first set of crosses (summarized in Supplementary Table S2).

Determining infection status

Individuals to be used in the crosses were established from single-mated females. The female was allowed to parasitize a host for $48 \mathrm{~h}$ and then the DNA was extracted. The presence of Wolbachia strains was confirmed by supergroup-specific primer pairs for each strain. Specific primer pairs for $w \mathrm{~B} 1$ were wsp-3_flkBF3/wsp-3_flkBR3, and for $w \mathrm{~B} 2$ were wsp-3_flkBF3A/wsp-3_flkBR3A (Raychoudhury

Table 1 Summary of the crosses done indicating which were incompatible (CI) and compatible (No CI)

\begin{tabular}{|c|c|c|c|}
\hline \multirow{2}{*}{$\begin{array}{l}\text { Male } \\
\text { Wolbachia polymorphism }\end{array}$} & \multirow{2}{*}{$\begin{array}{l}\text { Host } \\
\text { genotype }\end{array}$} & \multicolumn{2}{|r|}{ Female } \\
\hline & & $C I$ & No CI \\
\hline$(w \mathrm{~A} w \mathrm{~B} 1)_{\mathrm{UT} 1}$ & [UT1] & $\begin{array}{l}(w \mathrm{~A} w \mathrm{~B} 2)_{\mathrm{UT2}}[U T 2],(w \mathrm{~A})_{\mathrm{UT} 4}[U T 4], 0[C A], \\
0[U T 1],(w \mathrm{~A} w \mathrm{~B} 2)_{\mathrm{UT} 2}[U T 1]\end{array}$ & Self, $(w \mathrm{~A} w \mathrm{~B} 1 w \mathrm{~B} 2)_{\mathrm{UT} 3}[U T 3]$ \\
\hline$(w \mathrm{~A} w \mathrm{~B} 1)_{\mathrm{UT} 1}$ & {$[U T 1]^{\mathrm{a}}$} & $0[U T 1],(w \mathrm{~A} w \mathrm{~B} 2)_{\mathrm{UT} 2}[\mathrm{UT} 1]$ & Self \\
\hline$(w \mathrm{~A} w \mathrm{~B} 1)_{\mathrm{UT} 1}$ & {$[C A]$} & $0[C A]$ & Self, $(w \mathrm{~A} w \mathrm{~B} 2)_{\mathrm{UT} 2}[C A]$ \\
\hline$(w \mathrm{~A} w \mathrm{~B} 1)_{\mathrm{UT} 1}$ & [UT2] & $0[U T 2]$ & Self, $(w \mathrm{~A} w \mathrm{~B} 2)_{\mathrm{UT} 2}[U T 2]$ \\
\hline$(w \mathrm{~A} w \mathrm{~B} 2)_{\mathrm{UT} 2}$ & [UT2] & $\begin{array}{l}(w \mathrm{~A} w \mathrm{~B} 1)_{\mathrm{UT1}}[U T 1],(w \mathrm{~A})_{\mathrm{UT} 4}[U T 4], 0[C A], \\
0[U T 2],(w \mathrm{~A} w \mathrm{~B} 1)_{\mathrm{UT} 1}[U T 2]\end{array}$ & Self, $(w \mathrm{~A} w \mathrm{~B} 1 w \mathrm{~B} 2)_{\mathrm{UT} 3}[U T 3]$ \\
\hline$(w \mathrm{~A} w \mathrm{~B} 2)_{\mathrm{UT} 2}$ & {$[U T 2]^{\mathrm{a}}$} & $0[U T 2],(w \mathrm{~A} w \mathrm{~B} 1)_{\mathrm{UT} 1}[\mathrm{UT} 2]$ & Self \\
\hline$(w \mathrm{~A} w \mathrm{~B} 2)_{\mathrm{UT} 2}$ & {$[C A]$} & $0[C A],(w \mathrm{~A} w \mathrm{~B} 2)_{\mathrm{UT} 2}[C A]$ & Self \\
\hline$(w \mathrm{~A} w \mathrm{~B} 2)_{\mathrm{UT} 2}$ & [UT1] & $(w \mathrm{~A} w \mathrm{~B} 1)_{\mathrm{UT1}}[U T 1], 0[U T 1]$ & Self \\
\hline$(w \mathrm{~A} w \mathrm{~B} 1 w \mathrm{~B} 2)_{\mathrm{UT} 3}$ & [UT3] & $\begin{array}{l}(w \mathrm{~A} w \mathrm{~B} 1)_{\mathrm{UT} 1}[U T 1],(w \mathrm{~A} w \mathrm{~B} 2)_{\mathrm{UT} 2}[U T 2] \\
(w \mathrm{~A})_{\mathrm{UT} 4}[U T 4], 0[C A], 0[U T 3]\end{array}$ & Self \\
\hline$(w \mathrm{~A})_{\mathrm{UT} 4}$ & [UT4] & $0[C A], 0[U T 4]$ & $\begin{array}{l}\text { Self, }(w \mathrm{~A} w \mathrm{~B} 1)_{\mathrm{UT} 1}[U T 1],(w \mathrm{~A} w \mathrm{~B} 2)_{\mathrm{UT2}}[U T 2], \\
(w \mathrm{~A} w \mathrm{~B} 1 w \mathrm{~B} 2)_{\mathrm{UT}}[U T 3]\end{array}$ \\
\hline 0 & {$[C A]$} & & $\begin{array}{l}\text { Self, }(w \mathrm{~A} w \mathrm{~B} 1)_{\mathrm{UT} 1}[U T 1],(w \mathrm{~A} w \mathrm{~B} 2)_{\mathrm{UT} 2}[U T 2], \\
(w \mathrm{~A} w \mathrm{~B} 1 w \mathrm{~B} 2)_{\mathrm{UT} 3}[U T 3],(w \mathrm{~A})_{\mathrm{UT} 4}[U T 4]\end{array}$ \\
\hline 0 & [UT1] & & Self, (wA wB1) $)_{\mathrm{UT} 1}[U T 1], 0[U T 2], 0[U T 3], 0[U T 4]$ \\
\hline 0 & [UT2] & & Self, (wA wB2) $)_{\mathrm{UT} 2}[U T 2], 0[U T 2], 0[U T 3], 0[U T 4]$ \\
\hline 0 & [UT3] & & Self, (wA $w \mathrm{~B} 1 w \mathrm{~B} 2)_{\mathrm{UT} 3}[U T 3], 0[U T 2], 0[U T 3], 0[U T 4]$ \\
\hline 0 & [UT4] & & Self, $(w \mathrm{~A})_{\mathrm{UT} 4}[U T 4], 0[U T 2], 0[U T 3], 0[U T 4]$ \\
\hline
\end{tabular}

Self indicates crosses done within a particular strain.

${ }^{a}$ Indicates that the Wolbachia polymorphisms were re-introgressed into their native host genetic backgrounds. 
et al., 2009). To further confirm infection status, the amplified product of the supergroup B-specific ftsZ primer pair (Baldo et al., 2006b; Raychoudhury et al., 2009) was sequenced and checked for strain-specific polymorphisms, as $w$ NlonB1 and $w$ NlonB2 differ by a single base pair (C-A) in position 45 of the allele (Baldo et al., 2006b). The A supergroup Wolbachia was also similarly confirmed by PCR amplification and sequencing of the products of the A-specific ftsZ primer (Raychoudhury et al., 2009). The primer sequences, PCR conditions and sequencing protocol are given in Raychoudhury et al. (2009). To check for incomplete transmission of Wolbachia from the singlemated female to its progeny, some of the daughters were checked with specific primers. No loss of Wolbachia polymorphism was found during the crosses. Thus, the resulting crosses were not biased by the loss of the bacteria. To check for antibiotic curing of some of these strains the absence of any Wolbachia infections were tested with wspec primer pairs (Baldo et al., 2006b), which is sensitive for both the $A$ and $B$ infections. The presence of other endosymbionts, such as Cardinium, Rickettsia and Spiroplasma, has not been found in Nasonia. This is based on both bacterial group-specific PCR surveys and bacterial $16 S$ ribosomal sequencing (JH Werren, unpublished data).

\section{Statistical analysis}

CI in N. longicornis is expressed primarily as a reduction in the number of daughters and a slight increase in the number of sons in an incompatible cross (Bordenstein et al., 2001). As Nasonia has a haplodiploid mode of sex determination the zygotic death manifests itself in the diploid daughters, but not in the haploid sons. CI results in the loss of paternal chromatin, whereby most of the diploid zygotes are killed but some get transformed into males, which increases the number of sons in the progeny family size of an incompatible cross (Bordenstein et al., 2001). Thus, incompatible crosses in N. longicornis can be distinguished from a compatible cross by a reduction in the number of daughters and a slight increase in the number of sons. To test for statistical significance we used the non-parametric Mann-Whitney $U$ test (MWU) as employed in the program StatistiXL (BroadwayNedlands, Western Australia). The summary data for the crosses are indicated as means \pm s.e. of the family size.

\section{Results}

\section{Do (wAwB1) $)_{\mathrm{UT} 1}[U T 1]$ and $(w A w B 2)_{\mathrm{UT}}[\mathrm{UT2}]$ show bidirectional incompatibility in their native nuclear background?}

One of the major questions that we wish to answer is whether the two B infections are distinct incompatibility types. To test this we crossed these two strains, first, with the triple-infected males from the strain $(w \mathrm{~A} w \mathrm{~B} 1 w \mathrm{~B} 2)_{\mathrm{UT} 3}[U T 3]$ and then with each other. As Figure 1a indicates, there is significant CI between the females of these two strains and the males of the tripleinfected strain. The cross between the females of $(w \mathrm{~A} w \mathrm{~B} 1)_{\mathrm{UT1}}[U T 1]$ and $(w \mathrm{~A} w \mathrm{~B} 2)_{\mathrm{UT2}}[U T 2]$ and $(w \mathrm{~A} w \mathrm{~B} 1 w-$ $\left.{ }^{B 2}\right)_{\mathrm{UT} 3}$ [UT3] males produces progeny sizes that are significantly smaller (MWU, $U=746.0, P<0.001$ and $U=750.0, P<0.001$, respectively) when compared with the intra-strain controls indicating CI (Cross nos. 22 and
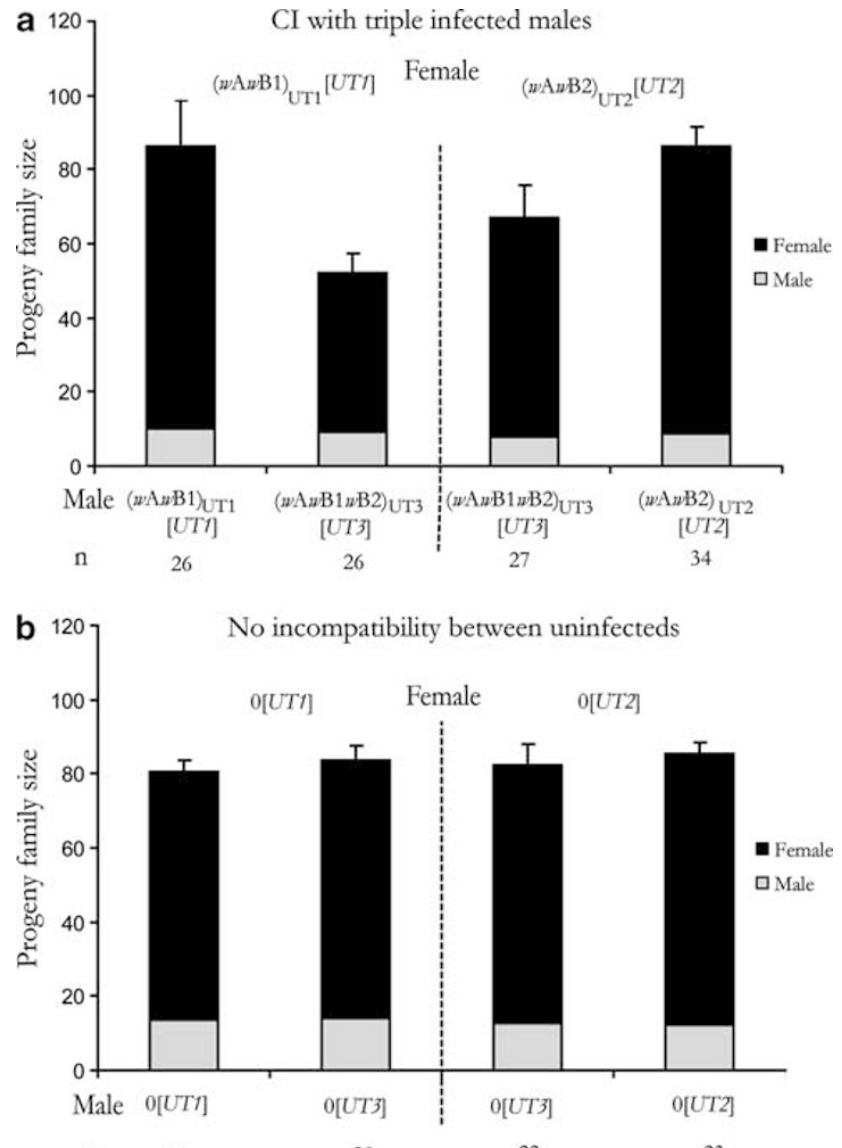

20

22

23

Figure 1 CI between double-infected females and triple-infected males. (a) CI seen between the females of the two B-infected strains and triple-infected ( $w \mathrm{~A} w \mathrm{~B} 1 w \mathrm{~B} 2)$ males. The columns on the left of the horizontal dotted line show CI between the males of $(w \mathrm{~A} w \mathrm{~B} 1 w \mathrm{~B} 2)_{\mathrm{UT3}}[U T 3]$ and females of $(w \mathrm{~A} w \mathrm{~B} 1)_{\mathrm{UT1}}[U T 1]$. The columns on the right show CI between the triple-infected males and $(w \mathrm{~A} w \mathrm{~B} 2)_{\mathrm{UT} 2}[U T 2]$-infected females. In both these sets of crosses the triple-infected males induce a significant reduction in the progeny family size when compared to the intra-strain controls (see text for details). (b) The reduction in progeny numbers is not due to nuclear genetic incompatibilities, as the same strains when cured of their Wolbachia show no significant reduction in progeny family size (MWU: $U=259.5, P=0.656$ and $U=280.5, P=0.536$, respectively).

23, Supplementary Tables S2 and S3). Thus, the two $B$ supergroup infections together produce a modification in the males, which cannot be rescued in the females by the individual $B$ infections. This indicates that the two $B$ infections represent different incompatibility types.

One explanation of the above results could be that the incompatibility observed is not due to the effects of the Wolbachia infections, but are strictly due to genetic incompatibilities between host strains (for example, they are cryptic species). To determine whether the incompatibilities are due to the effects of the bacteria and not due to host genetic background alone, we cured these strains of their Wolbachia infections by antibiotic treatment and repeated the crosses. The results (Figure $1 \mathrm{~b}$ and Table 1 , Cross nos. 24 and 25, Supplementary Tables S2 and S3) show that none of these crosses produces a significant reduction in the family size, indicating that the incompatibilities are produced due to effects of the bacteria and not by nuclear genetic incompatibilities between the 
strains. This supports the view that the two B infections consist of two distinct incompatibility types.

Based on the results of the cross with the males of the triple-infected strain $\left((w \mathrm{~A} w \mathrm{~B} 1 w \mathrm{~B} 2)_{\mathrm{UT3}}[U T 3]\right)$, we predicted that $(w \mathrm{~A} w \mathrm{~B} 1)_{\mathrm{UT} 1}$ and $(w \mathrm{~A} w \mathrm{~B} 2)_{\mathrm{UT} 2}$ should be bidirectionally incompatible (that is, each one not able to rescue the modification of the other). To test this we crossed the two strains (wAwB1) $)_{\mathrm{UT} 1}[U T 1]$ and (wA w$B 2)_{U T 2}$ [UT2] with each other (Figure 2a). The results indicate that there is partial bi-CI between these two strains. When compared with the controls, the females of (wA $w \mathrm{~B} 1)_{\mathrm{UT1}}[$ [UT1] can only partially rescue the modification produced by $(w \mathrm{~A} w \mathrm{~B} 2)_{\mathrm{UT} 2}[\mathrm{UT} 2]$ males (MWU, $U=693.0, \quad P<0.001)$. Similarly, females of $(w \mathrm{~A} w \mathrm{~B} 2)_{\mathrm{U}-}$ ${ }_{\mathrm{T} 2}$ [UT2] also partially rescue the modification produced by $(w \mathrm{~A} w \mathrm{~B} 1)_{\mathrm{UT1}}[U T 1]$ males (MWU, $\left.U=454.5, P<0.001\right)$ based on reduction in progeny family size. No significant reduction in family sizes could be detected when these crosses were repeated with cured strains (Figure 2b, Cross nos. 31 and 32, Supplementary Tables S2 and S3), showing that the effect is not due to any intrinsic nuclear genetic incompatibility between the host strains. Thus, the two B infections produce bi-CI against each other and represent two distinct incompatibilities, at least in their native nuclear genetic backgrounds.

\section{Do all the A supergroup infections have a similar incompatibility type?}

One of the causes of the bi-CI seen in the two B-infected strains could be due to the differences in their resident $A$ supergroup infections, even though each is identical in sequence at six strain typing genes (see Materials and methods). If the different $\mathrm{A}$ infections all represent distinct incompatibility types, then the bi-CI seen between these strains would be caused by these A infections and would be indistinguishable from the effects of the B Wolbachia, especially as these infections could not be separated from each other. If, on the other hand, the A infections share similar incompatibility types, then the double- and triple-infected females should be able to rescue the modification produced in males by the single A infection. To test whether the A infections present in the different strains have a similar incompatibility type we crossed males from the single A-infected strain with females of the triple-infected, as well as both the double-infected strains. As Figure 3 shows there is no significant reduction in progeny family size, indicating that multiple-infected females can all rescue the modification produced by the single A infection. In particular, females of the two doubleinfected strains, (wA wB1) $)_{\mathrm{UT1}}[U T 1]$ and $(w \mathrm{~A} w \mathrm{~B} 2)_{\mathrm{UT2}}[$ [UT2] can rescue modification of the males produced by $(w \mathrm{~A})_{\mathrm{UT} 4}[$ [UT4], as there are no significant reductions in progeny family sizes (MWU, $U=421.5, P=0.619$ and $U=340.0, P=0.162$, respectively). Similarly, the modification produced by the single A-infected males can also be rescued by triple-infected females (MWU, $U=36$, $P=1.0)$. This implies that either the $(w \mathrm{~A})_{\mathrm{UT} 4}[$ [UT4] strain is not producing any modification or all the $\mathrm{A}^{\prime} \mathrm{s}$ have similar or the same CI type with respect to the ability to rescue the modification produced by males of the single A-infected strain. To test the possibility that the $(w \mathrm{~A})_{\mathrm{UT} 4}[$ [UT4] strain does not produce any incompatibility, we crossed males from this strain with the
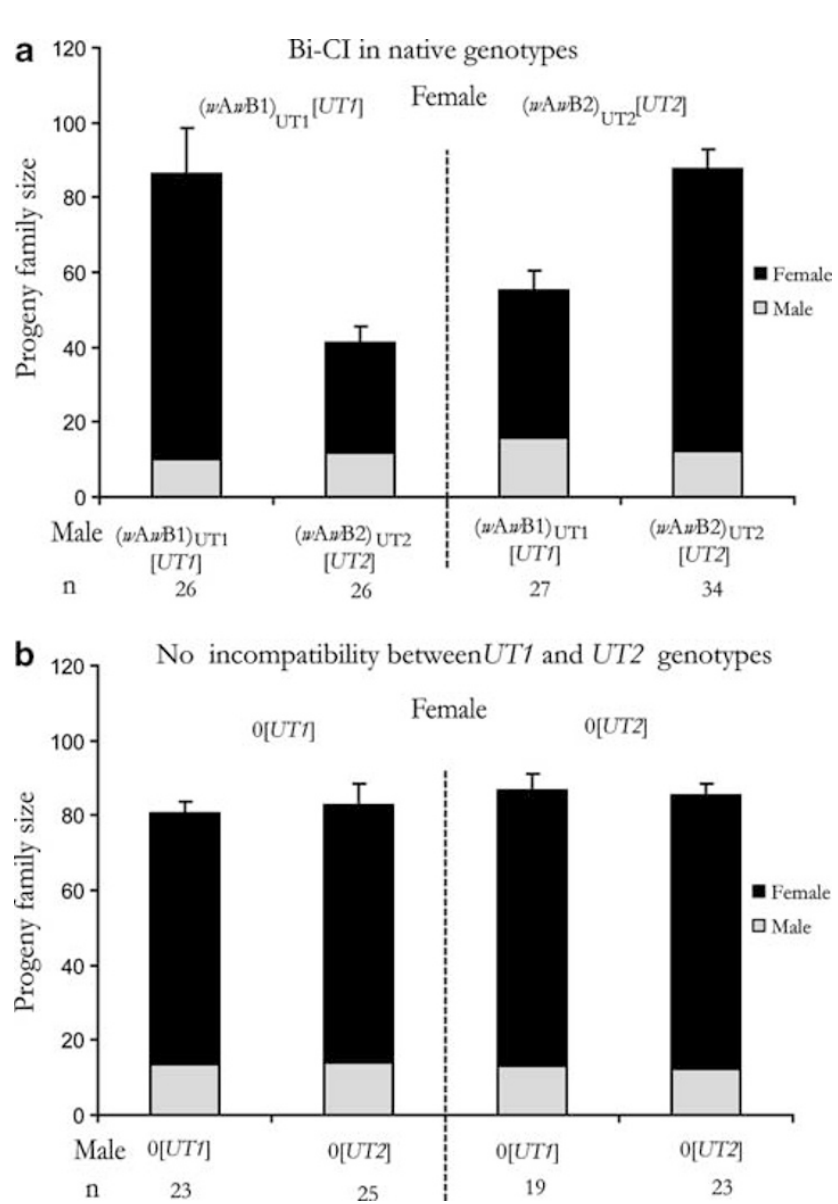

Figure 2 Bi-CI between the two double-infected strains. (a) Bi-CI seen between $(w \mathrm{~A} w \mathrm{~B} 1)_{\mathrm{UT} 1}$ and $(w \mathrm{~A} w \mathrm{~B} 2)_{\mathrm{UT} 2}$-infected strains in their own native host genotypes (see text for details). (b) The reduction in progeny numbers is not due to nuclear genetic incompatibilities, as the same strains when cured of their Wolbachia show no significant reduction in progeny family size. (MWU: $U=312.0, P=0.623$ and $U=238.0, P=0.635$, respectively).

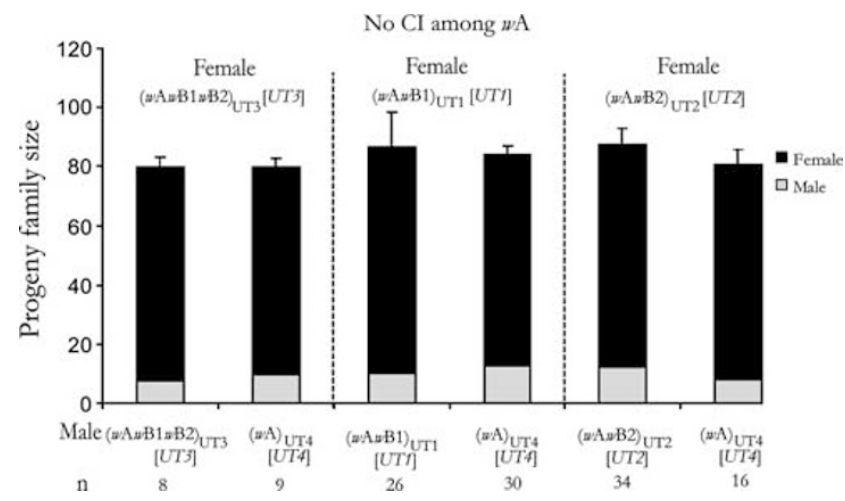

Figure 3 All the A supergroup infections share the same incompatibility type as the modification produced by the $(w \mathrm{~A})_{\mathrm{UT}}[$ [UT4]infected males can be rescued by all the other three infection polymorphisms. (MWU: $U=36, P=1.0, U=421.5, P=0.619$ and $U=340.0, P=0.162$, respectively).

Wolbachia-cured version of the same strain (0[UT4]), as well as with $0[C A]$ females. Results indicate that in both these sets of crosses the $(w \mathrm{~A})_{\mathrm{UT}}[$ [UT4] males do induce CI (Table 1, Cross nos. 20 and 21, Supplementary Tables S2 and S3). Thus, the most parsimonious explanation is that the bi-CI seen in the previous crosses, involving the 
two B infections, is due to differences in the B infection types, and not due to differences in the A infection.

\section{Do the $A$ and the two $B$ infections have different} incompatibility types?

One of the key questions about the Wolbachia infections in $N$. longicornis is whether they all represent different incompatibility types. We have already established that the two B infections are distinct incompatibility types as they produce bi-CI against each other (Table 1). Having established that all the A infections are similar in their incompatibility, we now asked whether this infection produces CI against the two B infections. As the individual infections could not be separated we used multiple Wolbachia-infected males against females from the single A-infected strain $\left((w \mathrm{~A})_{\mathrm{UT} 4}[U T 4]\right)$. Males from $(w \mathrm{~A} w \mathrm{~B} 1)_{\mathrm{UT} 1}[U T 1]$ and $(w \mathrm{~A} w \mathrm{~B} 2)_{\mathrm{UT} 2}[U T 2]$ strains would have their sperm modified by two different Wolbachia and males from $(w \mathrm{~A} w \mathrm{~B} 1 w \mathrm{~B} 2)_{\mathrm{UT}}[$ [UT3] would have three infections modifying their sperm but the female would only have the $w$ NlonA infections to rescue that modification. Therefore, if these are different incompatibility types there will be a significant reduction in the progeny family size owing to CI. As Figure 4a indicates, the females show significant CI with $(w \mathrm{~A} w \mathrm{~B} 1)_{\mathrm{UT1}}[U T 1]$ males (MWU, $U=380.0, P=0.002$ ), as well as with $(w \mathrm{~A} w \mathrm{~B} 2)_{\mathrm{UT2}}[U T 2]$ males (MWU, $\left.U=441.0, P<0.001\right)$. Moreover, there is also CI when the females of $(w \mathrm{~A})_{\mathrm{UT} 4}[$ UT4] are crossed with males from $(w \mathrm{~A} w \mathrm{~B} 1 w$ B2) ${ }_{\mathrm{UT3}}$ [UT3] (MWU, $\left.U=454.0, P<0.001\right)$. The uninfected control cross (Figure 4b, Cross nos. 15, 16 and 17, Supplementary Tables S2 and S3) shows that these results are not influenced by intrinsic host nuclear incompatibilities. Thus, the three infections, $w$ NlonB1, $w$ NlonB2 and $w$ NlonA, represent three different incompatibility types, at least in their native genetic backgrounds.

\section{Does the bi-Cl between (wAwB1) $\mathrm{UT1}$ and (wAwB2) UT2} change in an uniform genetic background?

A confounding factor during the expression of $\mathrm{CI}$ is the effect of host genetic background. Host genetic effects are known to moderate CI levels in diverse host taxa (Boyle et al., 1993; Bordenstein and Werren, 1998; McGraw et al., 2001; Ikeda et al., 2003; Kondo et al., 2005; Mouton et al., 2007). Therefore, to minimize the effects of different host backgrounds, we introgressed all these Wolbachia genotypes to a single genetic background by back-crossing them into the $0[C A]$ background for six generations (see Materials and methods: Introgression lines). When the crosses were repeated in this uniform genetic background the results (Figure $5 b$ ) indicated that bi-CI was transformed to unidirectional CI. Specifically, $(w \mathrm{~A} w \mathrm{~B} 2)_{\mathrm{UT} 2}[\mathrm{CA}]$ males show CI when crossed to $(w \mathrm{~A} w \mathrm{~B} 1)_{\mathrm{UT} 1}[C A]$ females (MWU, $U=385.0 . \quad P<0.001$ ) but not the other way around (MWU, $U=133.5$, $P=0.665)$. Thus, there is a pronounced effect of host genetic background on CI produced by $(w \mathrm{~A} w \mathrm{~B} 1)_{\mathrm{UT} 1}$ males but not by $(w \mathrm{~A} w \mathrm{~B} 2)_{\mathrm{UT} 2}$ males. One of the causes of the lack of $\mathrm{CI}$ on the part of $(w \mathrm{~A} w \mathrm{~B} 1)_{\mathrm{UT} 1}[C A]$ males could have been loss of one or both their Wolbachia infections. But the presences of both Wolbachia were confirmed by PCR (see Materials and methods) before and after the crosses were done. The other reason for this conversion
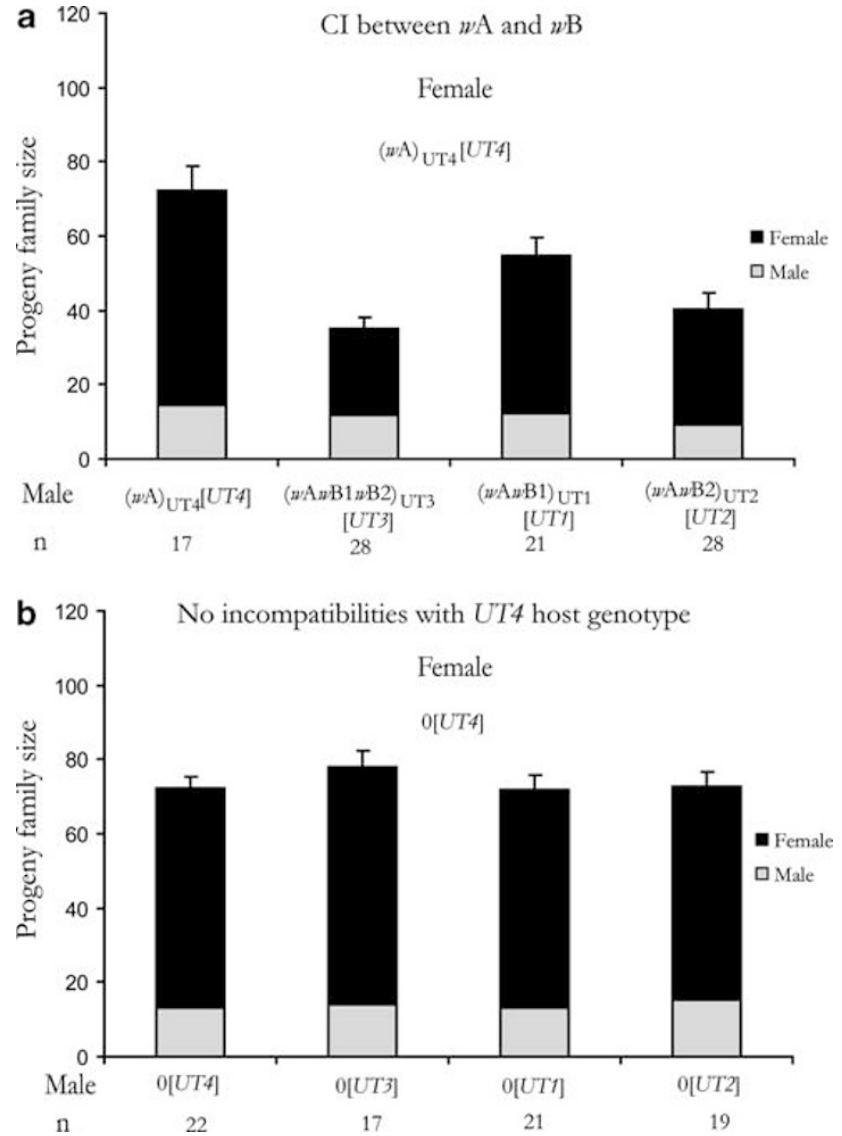

Figure 4 All the three Wolbachia in N. longicornis represent distinct incompatibility types. (a) The A and the two B supergroup infections all represent distinct incompatibility types. All the three multiply infected males produce $\mathrm{CI}$ against the single $w \mathrm{~A}$-infected females. The first column is the intra-strain control of the single A-infected strain $(w \mathrm{~A})_{\mathrm{UT} 4}[$ UT4]. The second, third and fourth column represents the progeny family size when the females of this strain were crossed with males from $(w \mathrm{~A} w \mathrm{~B} 1 w \mathrm{~B} 2)_{\mathrm{UT3}}$ [UT3], $(w \mathrm{~A} w \mathrm{~B} 1)_{\mathrm{UT} 1}[U T 1]$ and $(w \mathrm{~A} w \mathrm{~B} 2)_{\mathrm{UT} 2}[U T 2]$, respectively. There is significant reduction in the progeny family size in each of these crosses (see text for details). (b) The reduction in progeny number is not caused by host nuclear incompatibilities, as the same strains when cured of their Wolbachia show no significant reduction in progeny size. (MWU: $U=253, P=0.063, U=242.0, P=0.801$ and $U=227.0, P=0.651$, respectively).

of CI could be that the $(w \mathrm{~A} w \mathrm{~B} 1)_{\mathrm{UT} 1}$ males were not modifying sperm in this new host genetic background. We crossed the males of this strain with 0[CA] females and the results indicate that there is $C I$, and therefore modification of sperm (Cross no. 34, Supplementary Table S2 and S3).

Does the bi-Cl between (wAwB1) $)_{\mathrm{uT} 1}$ and (wAwB2) UT2 change in reciprocal genetic backgrounds?

One of the reasons why the $(w \mathrm{~A} w \mathrm{~B} 1)_{\mathrm{UT} 1}[C A]$ males failed to produce $\mathrm{CI}$ could be owing to an interaction between the new host genotype and the resident Wolbachia infections. This particular genotype was collected from California, although the native genotype of the (wAwB1) ${ }_{\mathrm{UT} 1}[$ [UT1] strain is from Utah (online Supplementary Table S1). Therefore, to investigate the host effect we reciprocally introgressed both these strains into the 

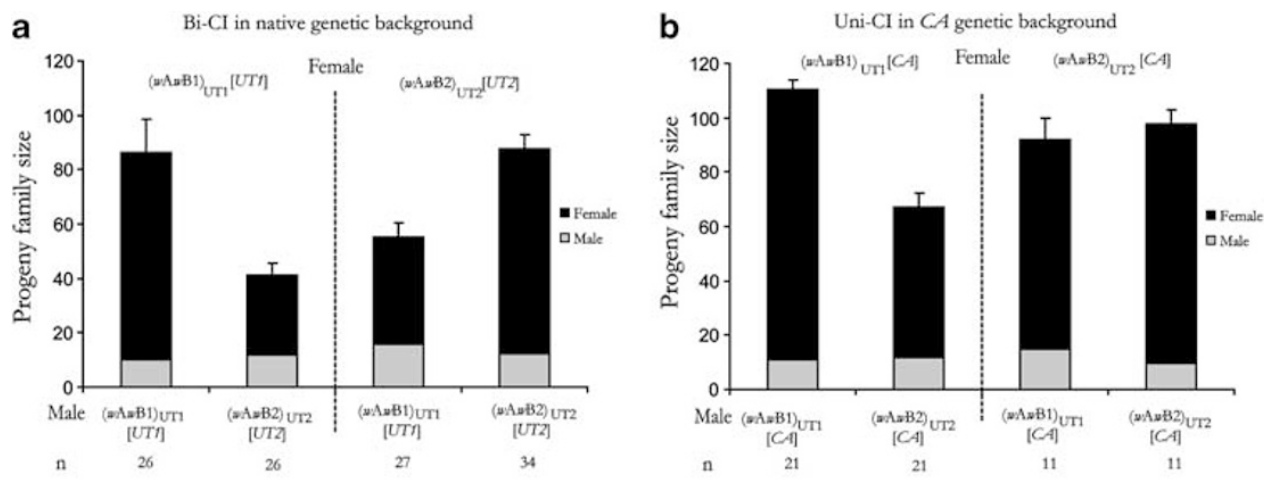

C
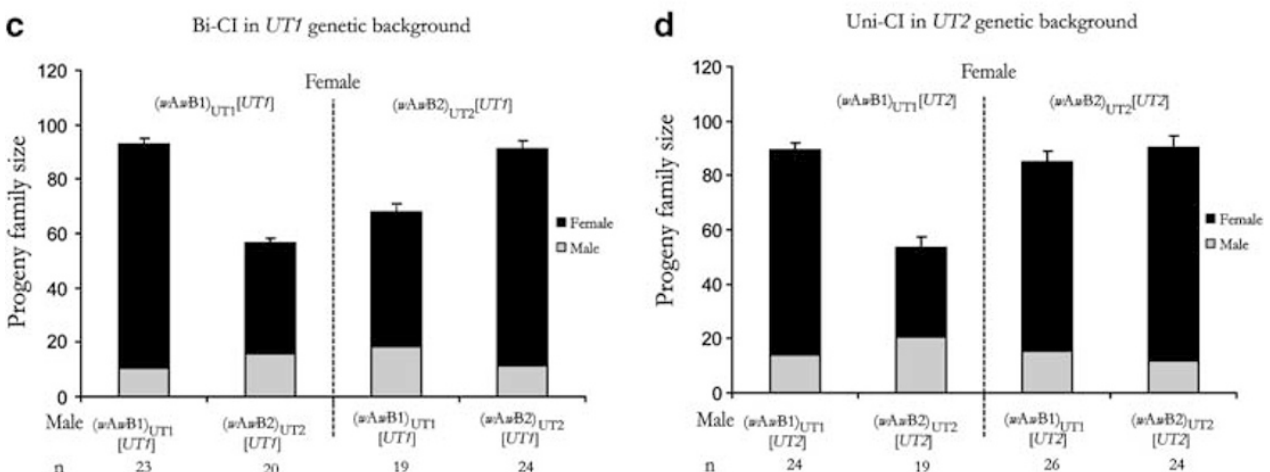

Figure $5 \mathrm{CI}$ between the two strains $(w \mathrm{~A} w \mathrm{~B} 1)_{\mathrm{UT} 1}$ and $(w \mathrm{~A} w \mathrm{~B} 2)_{\mathrm{UT} 2}$ in different host genetic backgrounds. (a) Bi-CI between $(w \mathrm{~A} w \mathrm{~B} 1)_{\mathrm{UT} 1}$ and $(w \mathrm{~A} w \mathrm{~B} 2)_{\mathrm{UT} 2}$ in their native host genetic background. (b) Uni-CI between (wAwB1) $\mathrm{UT11}$ and (wAwB2) $\mathrm{UT2}$ in the $C A$ genetic background. (c) $\mathrm{Bi}-\mathrm{CI}$ between $(w \mathrm{~A} w \mathrm{~B} 1)_{\mathrm{UT} 1}$ and $(w \mathrm{~A} w \mathrm{~B} 2)_{\mathrm{UT} 2}$ in the UT1 genetic background. (d) Uni-CI between ( $\left.w \mathrm{~A} w \mathrm{~B} 1\right)_{\mathrm{UT} 1}$ and $(w \mathrm{~A} w \mathrm{~B} 2)_{\mathrm{UT} 2}$ in the UT2 genetic background. Panel $\mathbf{a}$ is the same as Figure $2 \mathrm{a}$ and is repeated here for comparison.

native genotypes of each other. This was also done to establish whether re-introgressing the $(w \mathrm{~A} w \mathrm{~B} 1)_{\mathrm{UT} 1}$ strain back to its native genotype would enable the recovery of bi-CI. As Figure 5c indicates, there is bi-CI between these two strains in the UT1 genetic background. What has to be noted is that the $(w \mathrm{~A} w \mathrm{~B} 1)_{\mathrm{UT1}}[U T 1]$ is in its native genotype whereas the $(w \mathrm{~A} w \mathrm{~B} 2)_{\mathrm{UT} 2}$ strain has been introgressed into the former's host genotype (that is UT1). There is a significant reduction in family size when $(w \mathrm{~A} w \mathrm{~B} 2)_{\mathrm{UT} 2}[\mathrm{UT} 1]$ males were crossed to $(w \mathrm{~A} w \mathrm{~B} 1)_{\mathrm{UT1}}[U T 1]$ females (MWU, $U=459.0 . P<0.001$ ), as well as in the reciprocal cross (MWU, $U=430.0$, $P<0.001)$. In contrast, when the strain $(w \mathrm{~A} w \mathrm{~B} 1)_{\mathrm{UT} 1}$ [UT1] was introgressed into the native host genetic background of $(w \mathrm{~A} w \mathrm{~B} 2)_{\mathrm{UT} 2}$ (that is UT2), there was no bi-CI (Figure 5d, Table 1). Males from (wAwB2) $)_{\mathrm{UT} 2}[U T 2]$ produced a significant reduction in the progeny family size, when compared with the controls (MWU, $U=394.5$, $P<0.001)$. But no significant reduction in progeny family size was seen between the crosses involving (wA $w \mathrm{~B} 1)_{\mathrm{U}}$ ${ }_{\mathrm{T} 1}[U T 2]$ males and $(w \mathrm{~A} w \mathrm{~B} 2)_{\mathrm{UT}}[$ [UT2] females (MWU, $U=374.5, P=0.227)$. Thus, what is clear from all of these crosses is that males with wNlonB1 Wolbachia only induce CI when they have the UT1 genetic background. This has been established further by re-introgression to its native host genetic background. The $w$ NlonB2 Wolbachia, however, produces CI irrespective of the genetic background it is in.

\section{Discussion}

The two supergroup B infections in N. longicornis were documented to have diverged from each other approxi- mately 1.5 million years ago (Raychoudhury et al., 2009). Our results indicate that these two Wolbachia strains are bidirectionally incompatible in one genetic background while unidirectionally incompatible in others. The host genetic background has in the past been implicated to have a very significant role in the expression of CI (reviewed in Jaenike, 2009), and this study further demonstrates that host genotypes can determine whether CI is unidirectional or bidirectional. A similar effect was found between two strains of Culex mosquitoes (Sinkins et al., 2005), suggesting that host genetic alteration between bidirectional and uni-CI may be widespread.

Two distinct hypotheses can be put forward to explain the switch from bi-CI to uni-CI. The first hypothesis is that $w$ NlonB1 and $w$ NlonB2 are reciprocally incompatible in their native genetic backgrounds. However, modification of sperm by $w$ NlonB1 is 'repressed' in the two other genetic backgrounds (UT1 and CA), resulting in uni-CI. An alternative to the above hypothesis involves a more complex scenario of bacterial density changes causing incompatibility between the A and $\mathrm{B}$ infections. It posits that the $w$ NlonB1 infection does not cause CI with $w$ NlonB2, but rather, differential changes in the density between $w$ NlonA and $w$ NlonB2 causes the changes in CI. In this model, in the UT1 genetic background $w$ Nlon $A$ has its density increased and the $w$ NlonB1density decreased. This manifests itself as bi-CI between $(w \mathrm{~A} w \mathrm{~B} 1)_{\mathrm{UT} 1}$ and $(w \mathrm{~A} w \mathrm{~B} 2)_{\mathrm{UT} 2}$ strains. In the other two host genetic backgrounds that density of the $w$ NlonA is not increased and there is uni-CI with $w$ NlonB2 bearing males. However, this second hypothesis poses several problems. If the $w$ NlonB1 infection 
is not producing $\mathrm{CI}$, then, why is it being maintained? One of the explanations can be that it is a mutualist and is providing some benefits to the host. But this is not very apparent in the laboratory, as far as progeny number of the infected and cured host strains are concerned, because there is no significant difference between the two (MWU, $U=381.5, P=0.103$, Cross nos. 2 and 11, Supplementary Tables S2 and S3). It, however, could provide other benefits, such as protection against viruses, as has been found recently in D. melanogaster (Hedges et al., 2008; Teixeira et al., 2008). Another problem with the second hypothesis is that it cannot readily explain the occurrence of CI between $(w \mathrm{~A} w \mathrm{~B} 1 w \mathrm{~B} 2)_{\mathrm{UT} 3}[$ [UT3] males and $(w \mathrm{~A} w \mathrm{~B} 2)_{\mathrm{UT2}}[U T 2]$ females (Figure 1a and Table 1). If the $w$ NlonB1 is not involved in producing any incompatibility then the above cross should be compatible. Thus, the most likely explanation for the switch of CI from bidirectional to unidirectional remains the first hypothesis, which posits nuclear genetic 'repression' of sperm modification by $w$ NlonB1 in two different genetic backgrounds. Such a repression could be mediated through reduction in $w$ NlonB1 bacterial densities (for example, in testes). Although PCR results have established that none of the bacteria are lost in the different genetic backgrounds, quantitative PCR of the densities of these different Wolbachia strains can help to elucidate whether host genotype effects are mediated through changes in density of the different bacterial types.

The present study is also relevant to the debate concerning the potential role of Wolbachia in producing reproductive barriers between populations. Although controversial, Wolbachia has been suggested to promote speciation by preventing gene flow between infected and uninfected or differently infected populations (reviewed in Werren, 1998; Bordenstein, 2003). In Nasonia, Wolbachia has been shown to be a major contributor to reproductive incompatibility between $N$. longicornis and $N$. giraulti (Bordenstein et al., 2001). One of the ways that Wolbachia can promote speciation is by inducing bidirectional CI, where gene flow is stopped entirely or restricted substantially between populations infected with different CI causing Wolbachia. Various theoretical studies have shown that such an event is plausible under some conditions (Telschow et al., 2002, 2005a, b).

The present study shows that bi-CI is also dependant on host genotype. The $(w \mathrm{~A} w \mathrm{~B} 1)_{\mathrm{UT} 1}$ strain produces bi-CI only in one host genetic background (UT1) and not in the other two. Thus, bi-CI is a result of the interaction between the Wolbachia and host genotype. Therefore, the present study shows another novel way of producing bidirectional CI, where interactions between Wolbachia and host genotype together, and not singly, cause bidirectional CI. A question that follows from this observation is whether these Wolbachia are producing any substantial reduction in gene flow among $N$. longicornis populations? Various clues indicate that this is probably not true. First, the two double infections are present as polymorphisms along with the triple-infected strains. Raychoudhury et al. (2009) showed that two different infection polymorphism (that is, double and triple) can share the same mitochondrial haplotype, indicating loss of one of the B infections from the tripleinfected strains. Therefore, any genetic divergence that builds up from bi-CI between the two double-infected strains would be swamped by the individuals from the triple-infected strains, because the females of latter strain would be compatible with males from both the doubleinfected strains. Second, no significant pre-mating isolation could be detected between these strains when they were crossed. All the inter-strain crosses happened readily, indicating no significant behavioral isolation has evolved in these strains. Moreover, no F1 postzygotic incompatibility could be detected in these strains when cured of their respective Wolbachia infections (Supplementary Tables S2 and S3).

A further question that emerges is what maintains these infection polymorphisms? Basic theory indicates that triple-infected strains would replace all the doubleinfected polymorphisms (Vautrin et al., 2008), because triple-infected females are compatible with both the double-infected males, whereas, both the double-infected females are incompatible with the triple-infected males. However, incomplete transmission of Wolbachia can maintain infection polymorphisms via a balance between loss of triple infections owing to incomplete transmission and selection for them by CI (Baldo et al., 2008). Indeed, Raychoudhury et al. (2009) found in a survey of $N$. longicornis across the west coast of North America that the frequency of $w$ NlonB1wNlonA, $w$ NlonB2wNlonA and $w$ NlonB1wNlonB2wNlonA were 8,30 and $58 \%$, respectively, and that triple infections were associated with both of the major mitochondrial haplotypes. This indicates that the triple-infected strains likely predate divergence of the mitochondrial clades of N. longicornis, supporting the view that the double infections are derived by stochastic loss or competitive exclusion of one of the B infections.

One of the crucial facts that emerge from the present study is the importance of host-genetic influences on the expression of CI. But what is surprising is that this effect manifests itself even within a species, that is, within $N$. longicornis. Moreover, most of these strains come from one of the two major mitochondrial haplogroups in N. longicornis (Raychoudhury et al., 2009), which is found mainly in Utah (called the Great Basin haplotype whereas the other, found mainly in California, is called the West Coast haplotype). Thus, even within the same mitochondrial haplotype there is a pronounced effect of host nuclear genotype on CI. An example of switch from bi-CI to uni-CI has been previously reported by Sinkins et al. (2005) in Culex quienquefasciatus mosquitoes. They found that the bi-CI between two strains, Bei and Pel, was lost when the latter's genome was introgressed into the former's cytoplasm. Theoretical studies predict that host suppressors of incompatibility will be selected for (Rousset et al., 1991; Turelli, 1994; Koehncke et al., 2009) and there are recent examples of such repressors being found in nature (Hornett et al., 2006). Koehncke et al. (2009) showed theoretically that selection particularly favors the evolution of nuclear suppressors of sperm modification in males, because such genotypes are reproductively compatible with both infected and uninfected types. The apparent loss of sperm modification by the wNlonB1 Wolbachia in some host nuclear genotypes could be due to selection for a nuclear suppressor of $w$ NlonB1 sperm modification. How such a suppressor polymorphism is maintained and why it would be specific to the $w$ NlonB1 bacterium remains unclear. 


\section{Conflict of interest}

The authors declare no conflict of interest.

\section{Acknowledgements}

We thank John Jaenike and three anonymous reviewers for helpful comments on the manuscript. We also thank R Edwards for help in laboratory. This work was supported by US National Science Foundation (DEB0821936).

\section{References}

Baldo L, Ayoub NA, Hayashi CY, Russell JA, Stahlhut JK, Werren JH (2008). Insight into the routes of Wolbachia invasion: high levels of horizontal transfer in the spider genus Agelenopsis revealed by Wolbachia strain and mitochondrial DNA diversity. Mol Ecol 17: 557-569.

Baldo L, Dunning Hotopp JC, Jolley KA, Bordenstein SR, Biber SA, Choudhury RR et al. (2006b). Multilocus sequence typing system for the endosymbiont Wolbachia pipientis. Appl Environ Microbiol 72: 7098-7110.

Berticat C, Rousset F, Raymond M, Berthomieu A, Weill M (2002). High Wolbachia density in insecticide-resistant mosquitoes. Proceedings of the Royal Society of London Series B-Biological Sciences 269: 1413-1416.

Bordenstein SR (2003). Symbiosis and the origin of species. In: Bourtzis K, and Miller T (eds). Insect Symbiosis. CRC Press: New York, NY.

Bordenstein SR, O'Hara FP, Werren JH (2001). Wolbachiainduced incompatibility precedes other hybrid incompatibilities in Nasonia. Nature 409: 707-710.

Bordenstein SR, Uy JJ, Werren JH (2003). Host genotype determines cytoplasmic incompatibility type in the haplodiploid genus Nasonia. Genetics 164: 223-233.

Bordenstein SR, Werren JH (1998). Effects of A and B Wolbachia and host genotype on interspecies cytoplasmic incompatibility in Nasonia. Genetics 148: 1833-1844.

Bordenstein SR, Werren JH (2007). Bidirectional incompatibility among divergent Wolbachia and incompatibility level differences among closely related Wolbachia in Nasonia. Heredity 99: 278-287.

Bourtzis K, Dobson SL, Braig HR, O'Neill SL (1998). Rescuing Wolbachia have been overlooked. Nature 391: 852-853.

Boyle L, O'Neill SL, Robertson HM, Karr TL (1993). Interspecific and intraspecific horizontal transfer of Wolbachia in Drosophila. Science 260: 1796-1799.

Breeuwer JA, Werren JH (1990). Microorganisms associated with chromosome destruction and reproductive isolation between two insect species. Nature 346: 558-560.

Breeuwer JAJ, Werren JH (1995). Hybrid breakdown between two haplodiploid species: The role of nuclear and cytoplasmic genes. Evolution 49: 705-717.

Charlat S, Le Chat L, Merçot H (2003). Characterization of non-cytoplasmic incompatibility inducing Wolbachia in two continental African populations of Drosophila simulans. Heredity 90: 49-55.

Clark ME, Veneti Z, Bourtzis K, Karr TL (2003). Wolbachia distribution and cytoplasmic incompatibility during sperm development: the cyst as the basic cellular unit of $\mathrm{CI}$ expression. Mech Dev 120: 185-198.

Dunning-Hotopp JC, Clark ME, Oliveira DC, Foster JM, Fischer P, Muñoz Torres MC et al. (2007). Widespread lateral gene transfer from intracellular bacteria to multicellular eukaryotes. Science 317: 1753-1756.

Duron O, Labbé P, Berticat C, Rousset F, Guillot S, Raymond M et al. (2006). High Wolbachia density correlates with cost of infection for insecticide resistant Culex pipiens mosquitoes. Evolution 60: 303-314.
Frost CL, Fernández-Marín H, Smith JE, Hughes WO (2010). Multiple gains and losses of Wolbachia symbionts across a tribe of fungus-growing ants. Mol Ecol 19: 4077-4085.

Giordano R, O'Neill SL, Robertson HM (1995). Wolbachia infections and the expression of cytoplasmic incompatibility in Drosophila sechellia and D mauritiana. Genetics 140: 1307-1317.

Hedges LM, Brownlie JC, O'Neill SL, Johnson KN (2008). Wolbachia and virus protection in insects. Science 322: 702.

Hilgenboecker K, Hammerstein P, Schlattmann P, Telschow A, Werren JH (2008). How many species are infected with Wolbachia?-a statistical analysis of current data. FEMS Microbiol Lett 281: 215-220.

Hoffmann AA, Clancy D, Duncan J (1996). Naturally-occurring Wolbachia infection in Drosophila simulans that does not cause cytoplasmic incompatibility. Heredity 76: 1-8.

Hornett EA, Charlat S, Duplouy AM, Davies N, Roderick GK, Wedell $\mathrm{N}$ et al. (2006). 'Evolution of male-killer suppression in a natural population'. Plos Biol 4: 1643-1648.

Hornett EA, Engelstädter J, Hurst GD (2010). 'Hidden cytoplasmic incompatibility alters the dynamics of male-killer/ host interactions'. I Evol Biol 23: 479-487.

Ikeda T, Ishikawa H, Sasaki T (2003). Infection density of Wolbachia and level of cytoplasmic incompatibility in the Mediterranean flour moth, Ephestia kuehniella. J Invertebr Pathol 84: 1-5.

Jaenike J (2007). Spontaneous emergence of a new wolbachia phenotype. Evolution 61: 2244-2252.

Jaenike J (2009). Coupled population dynamics of endosymbionts within and between hosts. Oikos 118: 353-362.

Jaenike J, Dyer KA, Cornish C, Minhas MS (2006). Asymmetrical reinforcement and Wolbachia infection in Drosophila. Plos Biol 4: 1852-1862.

Jiggins FM, Hurst GD, Majerus ME (2000). Sex-ratio-distorting Wolbachia causes sex-role reversal in its butterfly host. Proc $R$ Soc Lond B Biol Sci 267: 69-73.

Koehncke A, Telschow A, Werren JH, Hammerstein P (2009). Life and death of an influential passenger: Wolbachia and the evolution of CI-modifiers by their hosts. Plos One 4: e4425.

Kondo N, Nikoh N, Ijichi N, Shimada M, Fukatsu T (2002b). Genome fragment of Wolbachia endosymbiont transferred to X chromosome of host insect. Proc Natl Acad Sci USA 99: 14280-14285.

Kondo N, Shimada M, Fukatsu T (2005). Infection density of Wolbachia endosymbiont affected by co-infection and host genotype. Biol Lett 1: 488-491.

Koukou K, Pavlikaki H, Kilias G, Werren JH, Bourtzis K, Alahiotisi SN (2006). Influence of antibiotic treatment and Wolbachia curing on sexual isolation among Drosophila melanogaster cage populations. Evolution 60: 87-96.

Laven H (1959). Speciation by cytoplasmic isolation in the Culex pipiens complex. Cold Spring Harbor Symp. Quant Biol 24: 166-173.

McGraw EA, Merritt DJ, Droller JN, O'Neill SL (2001). Wolbachia-mediated sperm modification is dependent on the host genotype in Drosophila. Proceedings of the Royal Society of London Series B-Biological Sciences 268: 2565-2570.

Montchamp-Moreau C, Ferveur JF, Jacques M (1991). Geographic distribution and inheritance of three cytoplasmic incompatibility types in Drosophila simulans. Genetics 129: 399-407.

Mouton L, Henri H, Charif D, Bouletrea M, Vavre F (2007). Interaction between host genotype and environmental conditions affects bacterial density in Wolbachia symbiosis. Biol Lett 3: 210-213.

O'Neill SO, Karr TL (1990). Bidirectional cytoplasmic incompatibility between conspecific populations of Drosophila simulans. Nature 348: 178-180.

Perrot-Minnot MJ, Guo LR, Werren JH (1996). Single and double infections with Wolbachia in the parasitic wasp Nasonia vitripennis: effects on compatibility. Genetics 143: 961-972. 
Raychoudhury R, Baldo L, Oliveira DC, Werren JH (2009). Modes of acquisition of Wolbachia : Horizontal transfer, hybrid introgression and co-divergence in the Nasonia species complex. Evolution 63: 165-183.

Raychoudhury R, Desjardins CA, Buellesbach J, Loehlin DW, Grillenberger BK, Beukeboom L et al. (2010a). Behavioral and genetic characteristics of a new species of Nasonia. Heredity 104: $278-288$.

Rigaud T, Antoine D, Marcade I, Juchault P (1997). The effect of temperature on sex ratio in the isopod Porcellionides pruinosus: Environmental sex determination or a by-product of cytoplasmic sex determination? Evol Ecol 11: 205-215.

Rousset F, Raymond M, Kjellberg F (1991). Cytoplasmic incompatibilities in the mosquito Culex pipiens-how to explain a cytotype polymorphism. J Evol Biol 4: 69-81.

Sinkins SP, Walker T, Lynd AR, Steven AR, Makepeace BL, Godfray HC et al. (2005). Wolbachia variability and host effects on crossing type in Culex mosquitoes. Nature 436: 257-260.

Stevens L, Giordano R, Fialho RF (2001). Male-killing,nematode infections, bacteriophage infection, and virulence of cytoplasmic bacteria in the genus Wolbachia. Annu Rev Ecol and Syst 32: 519-545.

Stouthamer R, Breeuwer JA, Hurst GD (1999). Wolbachia pipientis: microbial manipulator of arthropod reproduction. Ann Rev Microbio 53: 71-102.

Teixeira L, Ferreira A, Ashburner M (2008). The bacterial symbiont Wolbachia induces resistance to RNA viral infections in Drosophila melanogaster. Plos Biol 6: 2753-2763.
Telschow A, Hammerstein P, Werren JH (2002). The effect of Wolbachia on genetic divergence between populations: models with two way migration. Am Nat 160: S54-S66.

Telschow A, Hammerstein P, Werren JH (2005a). The effect of Wolbachia versus genetic incompatibilities on reinforcement and speciation. Evolution 59: 1607-1619.

Telschow A, Yamamura N, Werren JH (2005b). Bidirectional cytoplasmic incompatibility and the stable coexistence of two Wolbachia strains in parapatric host populations. J Theor Biol 235: 265-274.

Turelli M (1994). Evolution of incompatibility-inducing microbes and their hosts. Evolution 48: 1500-1513.

Vautrin E, Genieys S, Charles S, Vavre F (2008). Do vertically transmitted symbionts co-existing in a single host compete or cooperate? A modelling approach. J Evol Biol 21: 145-161.

Vavre F, Fleury F, Lepetit D, Fouillet P, Boulétreau M (1999). Phylogenetic evidence for horizontal transmission of Wolbachia in host-parasitoid associations. Mol Biol Evol 16: 1711-1723.

Werren JH (1997). Biology of Wolbachia. Annu Rev Entomol 42: 587-609.

Werren JH (1998). Wolbachia and Speciation. Oxford University Press: New York.

Werren JH, Baldo L, Clark ME (2008). Wolbachia: Master manipulators of invertebrate biology. Nat Rev Micro 6: 741-751.

Werren JH, Beukeboom LW (1998). Sex determination, sex ratios, and genetic conflict. Annu Rev Ecol Systs 29: 233-261.

Zabalou S, Apostolaki A, Pattas S, Veneti Z, Paraskevopoulos C, Livadaras I et al. (2008). Multiple rescue factors 14 within a Wolbachia strain. Genetics 178: 2145-2160.

Supplementary Information accompanies the paper on Heredity website (http://www.nature.com/hdy) 